STRUCTURAL SCIENCE CRYSTAL ENGINEERING MATERIALS

ISSN 2052-5206

Received 12 September 2018

Accepted 17 September 2018

Edited by A. J. Blake, University of Nottingham, England

Keywords: molecular crystals; phase transitions; single-crystal diffraction analysis.

\section{Crystalline transformers: more within than meets the eye}

\author{
Larry R. Falvello*
}

Department of Inorganic Chemistry and Aragón Materials Science Institute (ICMA), University of Zaragoza - CSIC, Pedro Cerbuna 12, Zaragoza, E-50009, Spain. *Correspondence e-mail: falvello@unizar.es

Studies of phase transitions in molecular crystals are becoming more commonplace as improvements in instrumentation and technique enable more efficient exploration of the behavior of samples with varying external conditions, usually temperature. This scientific commentary provides contextual background on this type of study, with reference to an article on transformations in a ferrocenylacetylide-gold(I) complex [Makal (2018). Acta Cryst. B74, 427-435].

From ancient civilizations to latter-day New Age communities, natural crystals have been thought to be sources of energy, renewal and any number of other endowments - this despite their appearance of resolute tranquillity. While science takes a less fanciful approach to crystal properties, modern methods with ångström-scale probes reveal that the staid external crystalline form conceals a world of motion that lies within. And from these motions arise the transitions whose study is coming of age.

In this issue of Acta Crystallographica Section B, Anna Makal reports a study of a pair of transitions in single crystals of ferrocenylacetylidetriethylphosphinegold(I) - synthetic, molecular crystals - and this carefully conducted study can serve as a window into the growing research area focused on transitions in such crystals.

The crystals that inspired much admiration in antiquity were extended solids emerald, for example, which was mined as early as 5000 years ago and thought by the ancient Egyptians to promote fertility and rebirth. These were natural crystals, left behind by the processes that shaped the substances found on the pristine Earth.

The motions of atoms in such crystals are understood in general terms and are intimately related to the symmetries of the crystalline state. Allowed modes have been catalogued and symmetry-descent pathways summarized, for example, in International Tables, Vol. A1 (2004) or on the Bilbao Crystallographic Server (Aroyo et al., 2006, 2011). These describe the motions that must initiate transformations in such crystals.

The crystalline material whose transformations are described by Makal in this issue of Acta Crystallographica Section B is a synthetic molecular solid. Being molecular, it is composed of robust chemical fragments whose internal binding forces are stronger than the intermolecular forces that hold the crystal together. And it is synthetic: Nature did not create the molecule, which is the product of synthetic chemistry. And it was the chemist who incited this molecule to form a crystalline solid.

The study of molecular crystals, the vast majority of which are synthetic, has an aspect that does not exist in the study of extended solids, in that the molecule has its own internal modes of motion, which add a complex factor to the modes that are proper to the crystal per se. So although we know that we cannot just describe atoms moving around in any which way within molecular crystals, in fact the possibilities for concerted motions in molecular crystals are ample.

In the study by Makal, the motion that engenders the transition is complex indeed, and is described in the article as a molecular gearworks. The nature of this motion raises intriguing questions about whether it can be fully concerted or whether it must be propagated from one or more starting points within the sample. What is certain is that it reveals the capacity of the crystal to undergo a complicated rearrangement without crumbling. 
The fact that a crystalline substance is synthetic has a more fundamental implication in that in these substances we are studying crystals that formed without any constraints on structure and energetics derived from the geological environments in which many crystals appeared in Nature. Most synthetic molecular crystals are prepared under relatively mild conditions and are not subjected to further perturbations before being studied. The structural consequences of intermolecular forces in molecular crystals can thus reasonably be accumulated into a knowledge-based context constructed from what is now a large number of studies of crystalline substances.

The experiment by Makal also underscores the need for careful exploration of systems that undergo such transformations. An intermediate phase was discovered at intermediate temperatures, and this phase and the corresponding insight that it provides about the global process, could easily have been missed without a detailed examination of the structure at fine temperature intervals. As it turned out, the symmetry of the intermediate has the expected group-subgroup relationships with both of the end phases.

Single crystals, and in particular molecular crystals, took on a new protagonism during the second half of the last century, as a result of their use in the analysis of molecular structures. The diffraction measurements needed for most structure analyses require an extended periodic structure; crystallizing the substance under study is the most straightforward way of satisfying that requirement. It did not take long for scientists to raise the question of studying the crystals themselves, for insight into the crystalline state and its intrinsic properties. Hamilton \& Ibers (1968) noted the possibilities in terms of two extremes; we may see the crystal 'simply as a device for holding the molecule down so that we can take a good look at it', or in their alternative view, 'it is effects associated with the crystalline order which are of primary interest, and the

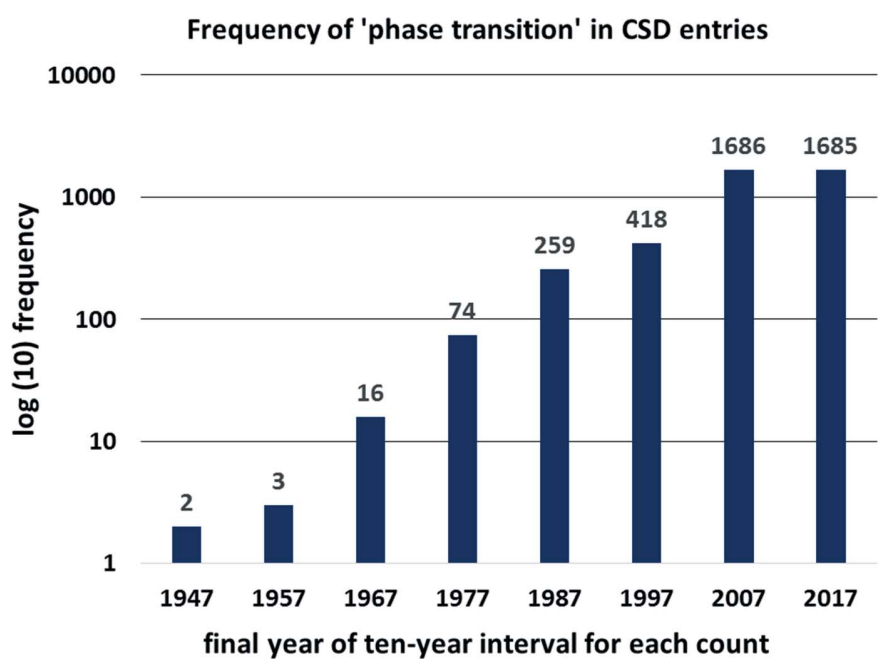

Figure 1

Frequency of occurrence of the text 'phase transition' in CSD entries for ten-year periods ending with the year indicated on the horizontal axis. The vertical scale is $\log (10)$. Only single-crystal analyses were considered. molecule may be looked upon as an evil to be avoided if at all possible.'

Early single-crystal studies of transitions in molecular crystals, such as the study of sodium potassium tartrate tetrahydrate (Rochelle salt; Ubbelohde \& Woodward, 1946), conducted before X-ray diffraction became an everyday tool of chemists, could involve quite specialized experimentation. Later, when single-crystal X-ray diffraction data were gathered using automated instruments with point detectors, time constraints still limited most structure analyses to a single data set at a single temperature. With the more widespread availability of area-detector-based instruments, it became feasible to study single crystals parametrically, conducting structure analyses under varying conditions of temperature. More recent advances in instrumentation are further enabling variable temperature and pressure studies on molecular crystals. A very coarse indicator of the developing trend in such studies using single-crystal diffraction is displayed in Fig. 1, a histogram of the number of entries in the Cambridge Structural Database (V5.39, updated to August, 2018; Groom et al., 2016) containing the text 'phase transition', published through ten-year periods ending in the year shown on the horizontal axis. The vertical axis is on a $\log (10)$ scale to permit the last two entries to fit into the same graphic with the rest.

The report by Makal also serves as a reminder that molecular crystals have been found to exhibit transformations of seemingly unlimited types, and that the growing body of such results is likely to be a signal that a complete understanding of a molecular crystalline substance requires an analysis of its transformations or of its lack thereof.

\section{Funding information}

The following funding is acknowledged: Ministerio de Ciencia, Innovación y Universidades (Spain) (grant No. MAT201568200-C2-1-P); Diputación General de Aragón (grant No. E11_17R).

\section{References}

Aroyo, M. I., Perez-Mato, J. M., Capillas, C., Kroumova, E., Ivantchev, S., Madariaga, G., Kirov, A. \& Wondratschek, H. (2006). Z. Kristallogr. Cryst. Mater. 221, 15-27.

Aroyo, M. I., Perez-Mato, J. M., Orobengoa, D., Tasci, E., de la Flor, G. \& Kirov, A. (2011). Bulg. Chem. Commun. 43, 183-197.

Groom, C. R., Bruno, I. J., Lightfoot, M. P. \& Ward, S. C. (2016). Acta Cryst. B72, 171-179.

Hamilton, W. C. \& Ibers, J. A. (1968). Hydrogen Bonding in Solids: Methods of Molecular Structure Determination. New York: W. A. Benjamin, Inc.

International Tables for Crystallography (2004). Vol. A1: Symmetry relations between space groups, edited by $\mathrm{H}$. Wondratschek \& U. Müller. London: Kluwer Academic Publishers.

Ubbelohde, A. R. \& Woodward, I. (1946). Proc. R. Soc. London Ser. $A, \mathbf{1 8 5}, 448-465$. 